\title{
Pectus excavatum: a rare cause of gastric subepi- thelial lesion
}

\author{
Sung Yong Han and Gwang Ha Kim
}

Department of Internal Medicine, Pusan National University School of Medicine, Busan, Korea

Received: October 30, 2016 Revised : November 19, 2016 Accepted: November 25, 2016

\section{Correspondence to}

Gwang Ha Kim, M.D.

Tel: +82-51-240-7869

Fax: +82-51-244-8180

E-mail: doco224@pusan.ac.kr
A 52-year-old woman presented with a gastric subepithelial lesion (SEL) on routine endoscopy. Upper endoscopy revealed a 5-cm SEL at the anterior wall of the upper body of the stomach (Fig. 1A). Neither a cushion sign nor a rolling sign appeared. Endoscopic ultrasonography revealed an extragastric lesion with sharp hyperechoic margins with posterior acoustic shadowing, which interrupted the detailed examination to observe inner structures (Fig. 1B). Abdominal computed tomography revealed a deformity in the left chest wall, with resultant compression of the stomach (Fig. 1C). Further inspection showed a sunken deformity in the left chest wall (Fig. 2). Final diagnosis was pectus excavatum, a congenital deformity of the anterior thoracic wall in which the sternum and rib cage grow internally.

\section{Conflict of interest}

No potential conflict of interest relevant to this article was reported.

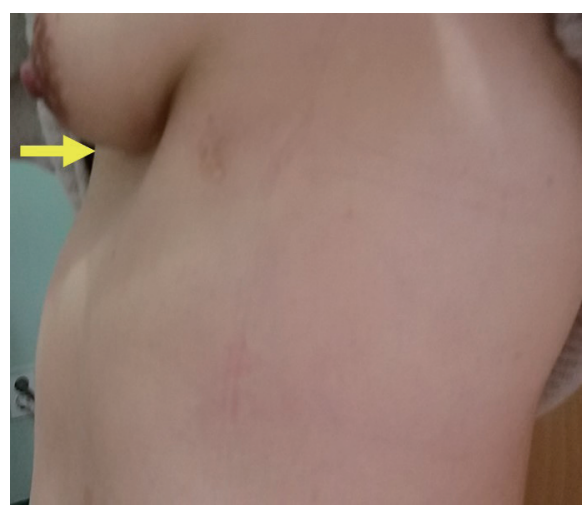

Figure 2. Inspection findings: a sunken appearance of the left chest wall (arrow).
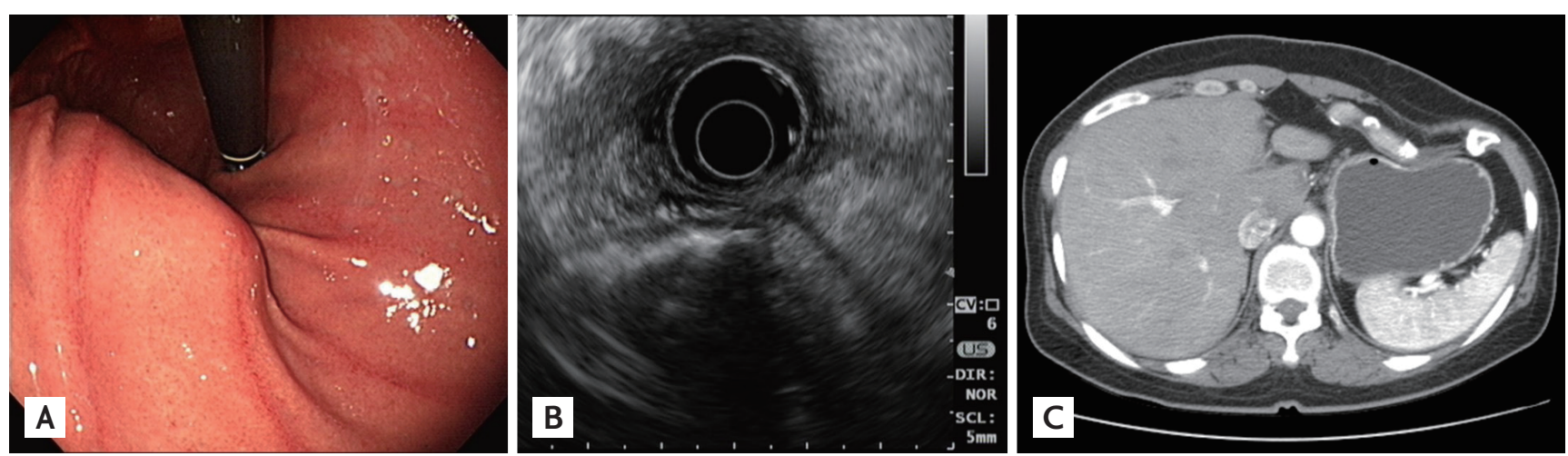

Figure 1. Image modality findings. (A) Upper endoscopy showing a 5-cm subepithelial lesion at the anterior wall of the upper body of the stomach. (B) Endoscopic ultrasonography showing an extragastric lesion with sharp hyperechoic margins with posterior acoustic shadowing. (C) Cross sectional scan of abdomen computed tomography showing a deformity in the left chest wall, with resultant compression of the stomach. 